\title{
Brother of regulator of imprinted sites inhibits cisplatin-induced DNA damage in non-small cell lung cancer
}

\author{
YANMEI ZHANG ${ }^{1 *}$, YONGFEI SONG ${ }^{1 *}, \mathrm{CHAO} \mathrm{LI}^{1}$, JUAN REN $^{1}$, \\ MENGDIE FANG ${ }^{1}$, JIANFEI FANG ${ }^{1,2}$ and XIAOJU WANG ${ }^{1}$ \\ ${ }^{1}$ Center for Molecular Medicine, Hangzhou Medical College, Hangzhou, Zhejiang 310012; \\ ${ }^{2}$ Department of Pathology, Institute of Cancer Research and Basic Medical Sciences of Chinese Academy of Sciences, \\ Cancer Hospital of University of Chinese Academy of Sciences, Zhejiang Cancer Hospital, Hangzhou, \\ Zhejiang 310022, P.R. China
}

Received September 29, 2019; Accepted May 27, 2020

DOI: $10.3892 / 01.2020 .12114$

\begin{abstract}
Cisplatin (DDP) chemotherapy is the primary modality of treatment for non-small cell lung cancer (NSCLC). However, due to the occurrence of DDP resistance, only a limited number of patients benefit from this treatment regimen. Brother of Regulator of Imprinted Sites (BORIS) is expressed elevated in NSCLC. Whether BORIS is involved in the DDP resistance of NSCLC is currently undetermined. The association between BORIS expression and overall survival rate of 156 patients with NSCLC who received DDP chemotherapy was analyzed in the present study. In order to investigate the function of BORIS in DDP chemotherapy, BORIS was silenced or overexpressed in four NSCLC cell lines. The cell viabilities, apoptosis and DNA damage induced by DDP were evaluated in these cell lines. In addition, the regulations of DNA repair genes were assessed, including POLH, ERCCl, BRCA1, MSH6 and XPA. The present study demonstrated that high BORIS expression was associated with decreased overall survival rate in patients with NSCLC who received DDP chemotherapy. The patients who benefited and went into remission following DDP therapy expressed a relatively low level of BORIS, suggesting the potential function of BORIS in DDP resistance. Cell experiments revealed that NSCLC cells that had a higher
\end{abstract}

Correspondence to: Dr Xiaoju Wang or Professor Yanmei Zhang, Center for Molecular Medicine, Hangzhou Medical College, 182 Tianmushan Road, Hangzhou, Zhejiang 310012, P.R. China

E-mail: wang.x.george@gmail.com

E-mail: yanmeizhang81@yahoo.com

*Contributed equally

Abbreviations: 5-FU, fluorouracil; A549/DDP, DDP resistant A549 cell line; BORIS, Brother of Regulator of Imprinted Sites; CTCF, CCCTC-binding factor; DDP, cisplatin; NSCLC, non-small-cell lung cancer; FACS, flow cytometry

Key words: chemotherapy response, cisplatin resistance, CTCF like factor, DNA repair, genome instability proliferation rate and resisted DDP treatment expressed a relatively higher level of BORIS. Knockdown of BORIS in NSCLC cells induced DNA damage; inhibiting cell proliferation and sensitizing cells to DDP treatment. In contrast, BORIS overexpression suppressed DDP-induced DNA damage. Notably, the mismatch repair factor mutS homolog 6 (MSH6) was regulated by BORIS, indicating its association with BORIS-associated DDP resistance in NSCLC. The findings of the present study suggest that BORIS suppresses DNA damage and promotes the progression of NSCLC and DDP resistance. The present study indicates the potential application of BORIS in NSCLC therapy and prognosis.

\section{Introduction}

Non-small cell lung cancer (NSCLC) accounts for $~ 80 \%$ of all types of lung cancer and was reported the leading cause of cancer-associated mortality worldwide in $2016(1,2)$. Cisplatin (DDP)-based chemotherapy regimens are recommended as the standard treatment modalities for NSCLC; however, only $\sim 20 \%$ of patients respond to the treatment $(3,4)$.

Brother of Regulator of Imprinted Sites (BORIS, also known as CCCTC-binding factor like, the paralogue of CCCTC-binding factor (CTCF), is abnormally expressed in the majority or different types of cancer and is therefore considered as a potential therapeutic target for breast, lung and cervical carcinoma (5-7). However, the functions of BORIS in carcinoma remain unknown. In our previous study (8), it was revealed that BORIS suppressed apoptosis and resisted fluorouracil (5-FU) treatment in colorectal cancer. Since 5-FU or DDP induces DNA damage and apoptosis of carcinoma cells (9-11), BORIS may induce resistance to DDP therapy. Based on the prevalent expression of BORIS and the high incidence of DDP resistance in NSCLC $(4,12)$, it is worth investigating whether BORIS contributes to DDP resistance in NSCLC.

Studies on platinum resistance in different types of cancer, such as ovarian cancer, lung cancer and breast cancer have revealed multiple complex resistance mechanisms, including the decreased accumulation of platinum, a decline in DDP-DNA adduct levels and an increase in DNA damage repair $(9,13,14)$. 
On the other hand, cancer cells are also able to correct intrinsic pathways, such as the DNA repair system (including DNA excision and mismatch repair systems) and apoptosis to defend against environmental toxins for survival $(9,14)$. It has been reported that aberrant activation of DNA repair pathways of NSCLC contributes to DDP resistance (15-18).

The presents study aimed to investigate whether BORIS promoted NSCLC cell proliferation and protected NSCLC cells from being injured by DDP. BORIS was used as the candidate target for NSCLC therapy to decrease DDP resistance.

\section{Materials and methods}

Cell culture and transfection. Cell lines K562, H1299, A549 and H460 were purchased from the American Type Culture Collection. In the three lung cancer cell lines, H1299 lacks the expression of p53 protein. A549 and H460 cells are detectable for p53 (19). A549/DDP (DDP-resistant A549 cell line) was purchased from Shanghai ExCell Biology, Inc. Cells were cultured in RPMI 1640 medium containing $10 \%$ heat-inactivated fetal bovine serum (FBS; Gemini Company, https://www.gembio.com/products) at $37^{\circ} \mathrm{C}$ and in a humidified atmosphere containing $5 \% \mathrm{CO}_{2}$ for 1 week.

Lipofectamine $^{\circledR} 3000$ reagent (Thermo Fisher Scientific, Inc.) was used to transfect pBORIS plasmids purchased from OriGene Technologies Inc.. The empty vector control was constructed in our previous study (7). According to the requirement of Lipofectamine ${ }^{\circledR} 3000,70 \%$ confluent cells were prepared for the transfection. Plasmid DNA $(1 \mu \mathrm{g})$ was transfected per well into6-well plates. Plasmid (50 ng) was transfected per well into 96-well plates. Lipofectamine RNAiMAX (Thermo Fisher Scientific, Inc.) was used to knockdown genes according to the manufacturer's instructions. Two siRNAs (siRNA-1 and siRNA-2) that targeted BORIS mRNA were used to knockdown BORIS. All small interfering RNAs (siRNA) were chemically synthesized by Xiang Yin Biotechnology Co., Ltd. (Table SI). The DDP treatment on transfected cells were performed one day after transfection.

Reverse transcription-quantitative $(R T-q)$ PCR. Cells $\left(5 \times 10^{5}\right)$ were harvested from cell culture plates. RNA was extracted using TRIzol ${ }^{\circledR}$ reagent (Thermo Fisher Scientific, Inc.) and ethanol precipitation. Samples were quantified using the NanoDrop 2000 system (Thermo Fisher Scientific, Inc.) and proceeded to reverse transcription. The kit (cat. no. CW0741) used for reverse transcription was purchased from cwbiotech Beijing (https://www.cwbiotech.com/home.html). Subsequently, gene expression levels were quantified using SYBR Green based qPCR (cwbiotech Beijing). The thermocycling conditions were as follow: Initial denaturation at $95^{\circ} \mathrm{C}$ for $3 \mathrm{~min} ; 45$ cycles of denaturation at $95^{\circ} \mathrm{C}$ for $10 \mathrm{sec}$, annealing at $60^{\circ} \mathrm{C}$ for $10 \mathrm{sec}$, elongation at $72^{\circ} \mathrm{C}$ for $30 \mathrm{sec}$; and afinal extension at $72^{\circ} \mathrm{C}$ for $30 \mathrm{sec}$. Amplification and signal capture were performed by Bio-Rad CFX connect real-time system (Bio-Rad Laboratories). GAPDH and $\beta$-actin were used as the internal control genes to evaluate the expression levels of the candidate genes. The primer sequences used for qPCR are presented in Table SII.
Western blotting. Total protein was extracted from collected samples using RIPA buffer (Beyotime Institute of Biotechnology) supplemented with phenylmethylsulfonyl fluoride (cat. no. CAS 329-98-6; Sigma-Aldrich; Merck KGaA) and protease inhibitor cocktail (cat. no. 04693159001; Roche Applied Science). Total protein was quantified using the bicinchoninic acid kit (cat. no. P0012S; Beyotime Institute of Biotechnology) and $30 \mu \mathrm{g}$ protein/lane was separated via $10 \%$ SDS-PAGE gel. The separated proteins were subsequently transferred onto nitrocellulose membranes and blocked with $5 \%$ skimmed milk for $1 \mathrm{~h}$ at room temperature. The membranes were incubated with BORIS polyclone primary antibody (cat. no. Ab126778; Abcam) overnight at $4^{\circ} \mathrm{C}$. The BORIS antibody was diluted by $0.1 \%$ in $2 \%$ skimmed milk. Following the primary incubation, membranes were incubated with secondary antibody conjugated with HRP (cat. no. DW-0990; Dawen Biotechnology Co., Ltd., www.dawenbio.com) for $2 \mathrm{~h}$ at room temperature, which was diluted by $0.05 \%$ in $2 \%$ skimmed milk and used for subsequent experimentation. Protein blots were detected using the Advansta ECL system (cat. no. K-12043-D10; Advansta Inc., https://advansta.com) and visualized using the Bio-Rad chemidoc XRS+ system (Bio-Rad Laboratories, Inc.).

Cell viability analysis and colony formation assay. For the cell viability assays, 2,000 cells were seeded onto 96 well-plates/well. Cells were incubated with MTT (500 $\mu \mathrm{g} / \mathrm{ml}$, cat. no. M2128, Sigma-Aldrich; Merck KGaA) at $37^{\circ} \mathrm{C}$ for $4 \mathrm{~h}$ in the dark. Following the MTT incubation, the purple formazan crystals were dissolved using $100 \mu \mathrm{l}$ dimethyl sulfoxide for $15 \mathrm{~min}$ at room temperature, and viability was subsequently analyzed at a wavelength of $490 \mathrm{~nm}$, using a BioTeck Synergy 2 plate reader system (http://www.mtxlsi. com/bio-tek-synergy-2.htm).

For the colony formation assays, treated cells were seeded onto 6 well plates and fixed with $4 \%$ formaldehyde for $20 \mathrm{~min}$ at $37^{\circ} \mathrm{C}$, and subsequently rinsed twice with PBS. Cells were stained with $0.5 \%$ crystal violet for $30 \mathrm{~min}$ at room temperature. Images were capturedusing white light channel in Bio-Rad chemidoc XRS+ system (Bio-Rad Laboratories, Inc.).

Flow cytometry (FACS) assay. The Alexa Fluor 488 Annexin V/Dead Cell Apoptosis kit (cat. no. V13241) was obtained from Thermo Fisher Scientific, Inc. Following treatment with DDP, $\sim 10^{6}$ cells were harvested and washed twice in cold PBS. According to the manual, $1 \mathrm{X}$ annexin binding buffer was used to dilute the Annexin V and propidium iodide (PI). Cells were then resuspended and incubated in Annexin V/PI working buffer at room temperature for $15 \mathrm{~min}$ in the dark. Subsequently, cells were stored in the dark and on ice. FACS was performed using a FACSCalibur (BD Biosciences) to detect apoptotic cells. Data analysis was performed using FlowJo software (version no. 7.6.1; FlowJo LLC).

Immunofluorescence and TUNEL assay. Cells mounted on coverslips were prepared for treatments. After treatments cells were fixed with $4 \%$ formaldehyde for $20 \mathrm{~min}$ at $37^{\circ} \mathrm{C}$ and subsequently permeabilized with $0.3 \%$ Triton $\mathrm{X}-100$ for $10 \mathrm{~min}$ at room temperature. Cells were subsequently blocked with $1 \%$ bovine serum albumin (BSA; Sangon Biotech Co., Ltd.) 
for $30 \mathrm{~min}$ at room temperature. Cells were incubated with primary antibody (1:200 in 1\% BSA) overnight at $4^{\circ} \mathrm{C}$. Cells were washed three times with PBS and subsequently incubated with secondary antibodies conjugated with tetramethyl rhodamine isothiocyanate (TRITC) or fluorescein-5-isothiocyanate (FITC; 1:200 in 1\% BSA) for $1 \mathrm{~h}$ at room temperature. Cell nuclei were stained using DAPI $(0.5 \mu \mathrm{g} / \mathrm{ml})$ at room temperature for $5 \mathrm{~min}$. Coverslips were re-washed four times and visualized using a Leica fluorescence microscope (magnification, x20). BORIS primary antibody (cat. no. Ab126778) was purchased from Abcam and $\gamma \mathrm{H} 2 \mathrm{AX}$ antibody (cat. no. 05-636) was purchased from EMD Millipore. Primary antibodies were diluted 1:200 in 1\% BSA. Flag antibody (cat. no. R1180B) was purchased from OriGene Technologies, Inc. FITC conjugated rabbit antibody (cat. no. DW-GAR001, www. dawenbio.com) and TRITC conjugated mouse antibody (cat. no. DW-A0521, www.dawenbio.com) were purchased from Dawen Biotechnology Co., Ltd..

TUNEL apoptosis detection kit (FITC) was bought from Shanghai Yeasen Biotechnology Co., Ltd.. The TUNEL reaction system was incubated at $37^{\circ} \mathrm{C}$ for $2 \mathrm{~h}$ in the dark. The cells were washed twice with PBS for 5 min. DAPI $(1: 6,000)$ was used to stain the nucleus in a dark room for $5 \mathrm{~min}$ in room temperature. After 2 washes with PBS, coverslips were sealed with glycerol and mounted onto slides. A Leica fluorescence microscope was used to visualize the cells and more than three scopes of images were captured by $20 x$ objective lens for further analysis.

RNA-sequencing (RNA-seq) analysis. RNA-seq data and the clinical information for patients with NSCLC treated with DDP were downloaded from The Cancer Genome Atlas (TGCA) data portal and manually curated (11) (Table SIII). Clinical characteristics for the lung cancer cases $(n=156)$ include age, sex, ethnicity, BORIS expression [fragments per kilobase of exon model per million reads mapped (FPKM)], clinical-stage (Tumor-Node-Metastasis; TNM) (3) and survival span. Treatment outcomes were recorded for 23 patients (Table SIV). Cancer tissues from 16 of the 23 patients were collected and analyzed before DDP treatment, which were shown to be 'prospective' in the present study. Cancer tissues from 7 of the 23 patients were collected and analyzed after DDP treatment, which were shown to be 'retrospective' in the present study (Table I). Survival probabilities of 156 NSCLC patients were evaluated when the patients were divided into two groups using a cut-off value of 0.0035 (FPKM).

Statistical analysis. Lung cancer classification for stagingwas referenced to worldwide standard (20). BORIS expression differences between patient groups were analyzed using the $\chi^{2}$ test (Table I). The survival probabilities of investigated patients were analyzed via the Kaplan-Meier method and compared using the log-rank test. BORIS expression differences between NSCLC cell lines were analyzed by one-way ANOVA followed Tukey post-hoc test. All experiments were performed in triplicate. Statistical analysis was performed using SPSS 24.0 software (IBM Corp.) or GraphPad Prism software (version 5.0; GraphPad Software, Inc.). Statistical differences were analyzed by paired Student's t-test and presented as the mean \pm standard deviation. $\mathrm{P}<0.05$ was considered to indicate a statistically significant difference.

\section{Results}

Patients with NSCLC with higher BORIS expression have a shorter survival span following DDP therapy. A total of 156 patients with NSCLC who received DDP treatment were extracted from TCGA (http://www.tcga.org/) (Tables I, II and SIII). The median of BORIS expression among the patients was 0.0035 (FPKM), which was used as a cut-off to study the association between BORIS and the overall survival rate in the present study. A higher BORIS expression level was significantly associated with a short survival span ( $\mathrm{P}=0.019$; Fig. 1A). In addition, BORIS expression levels in female patients were significantly lower than male patients. It is suggested that BORIS may be induced at high levels in male patients (Table I). BORIS expression in 23 cases with records of treatment outcomes was analyzed (Table SIV). BORIS expression data were extracted from The Cancer Genome Atlas database (http://www.tcga.org). Though no paired tissues were collected from the same patient before and after DDP therapy, BORIS levels declined from an average of 0.0071 FPKM in the prospective tissues to an average of 0.0027 FPKM in retrospective tissues (Fig. 1B). These data suggested that BORIS expression was associated with the mortality rate of NSCLC upon DDP chemotherapy.

BORIS expression is positively associated with NSCLC cell proliferation. Next, the proliferation rates of a panel of lung cancer cell lines was studied, which revealed a positive association between BORIS expression levels and cell proliferation (Fig. 1C and D). All cell lines tested demonstrated a statistically significant difference (Fig. 1C). Among the cell lines that were assessed, human pulmonary alveolar epithelial cell line (HPAEpiC) was a normal cell line. HPAEpiC did not express BORIS, suggesting that BORIS may just function in cancer cells. In order to further investigate its effect on cell proliferation, BORIS was silenced or overexpressed in NSCLC cell lines. A549/DDP cells express a relative higher BORIS compared with A549 (Fig. 1C). Next, two siRNAs targeting BORIS generated a similar knockdown efficiency with $>50 \%$ decrease (Table SI and Fig. 2D). The knockdown efficiencies of siRNA-1 were all $>70 \%$ in A549 and H460 cell lines (Fig. S1); therefore, in the present study, BORIS siRNA-1 was used for subsequent experimentation. BORIS knockdown suppressed the colony formation of NSCLC cells and induced the expression of $p 21$, a cyclin-dependent kinase inhibitor (Fig. 2A and B). It has previously been demonstrated that elevated expression of $p 21$ induces cell cycle arrest (21-23). Likewise, overexpression of BORIS promoted the proliferation of NSCLC cells and inhibited the expression of $p 21$ in H1299 and A549 cells (Fig. 2B and C). Among the investigated NSCLC cell lines in the present study, BORIS was highly expressed in H1299 cells and expressed at low levels in A549 cells. Thus, the regulations of p21 by BORIS was investigated in these two cell lines, which express different BORIS levels (Fig. 1C). As BORIS knockdown inhibited cell cycles of NSCLC cells, the genome stability was investigated by assessing $\gamma \mathrm{H} 2 \mathrm{AX}$, which is sensitive to DNA damage (24). BORIS was silenced by either siRNA-1 or siRNA-2 and the knockdown efficiencies were verified via western blotting and RT-qPCR analyses. The results demonstrated that BORIS 
Table I. Characteristics of 156 patients with NSCLC.

\begin{tabular}{|c|c|c|c|}
\hline Characteristic & High BORIS expression, FPKM $>0.0035, \mathrm{n}$ & Low BORIS expression FPKM $<0.0035, \mathrm{n}$ & P-value \\
\hline Sex & & & 0.0011 \\
\hline Male & 57 & 37 & \\
\hline Female & 21 & 41 & \\
\hline Race & & & 0.75 \\
\hline White & 63 & 59 & \\
\hline Black & 5 & 6 & \\
\hline Asian & 0 & 1 & \\
\hline Not reported & 11 & 11 & \\
\hline Age, years & & & $>0.999$ \\
\hline$>62$ & 38 & 39 & \\
\hline$\leq 62$ & 38 & 39 & \\
\hline Stage & & & 0.35 \\
\hline I & 17 & 14 & \\
\hline II & 41 & 37 & \\
\hline III & 16 & 23 & \\
\hline IV & 2 & 4 & \\
\hline Not reported & 2 & 0 & \\
\hline
\end{tabular}

Sex, ethnicity, age and stage of the 156 patients with NSCLC were grouped according to the BORIS expression level. The average level of BORIS in the 156 patients with NSCLC was 0.0035 FPKM. Data were assessed using the $\chi^{2}$ test. BORIS, Brother of Regulator of Imprinted Sites; NSCLC, non-small cell lung cancer; FPKM, fragments per kilobase of exon model per million reads mapped.

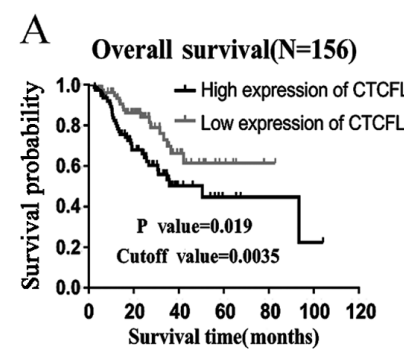

C

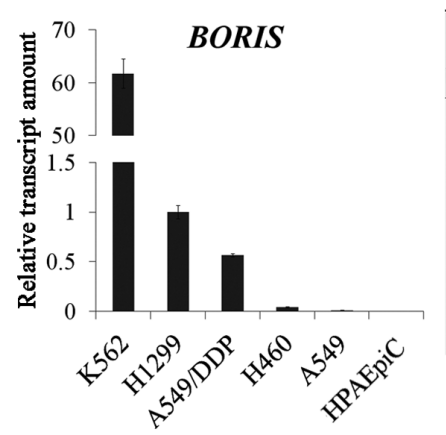

B Tissue collection:

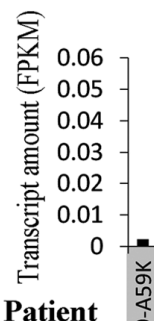

Patient

Response stable

\begin{tabular}{|c|c|c|c|c|}
\hline \multicolumn{5}{|c|}{$\begin{array}{l}\text { Significant Difference of BORIS Expression } \\
\text { between each NSCLC cell lines (Adjusted P value) }\end{array}$} \\
\hline & H1299 & A549/DDP & $\mathrm{H} 460$ & A549 \\
\hline K562 & 0.0006 & 0.0006 & 0.0005 & 0.0005 \\
\hline H1299 & & 0.0207 & 0.0044 & 0.0032 \\
\hline A549/DDP & & & 0.0001 & 0.0001 \\
\hline $\mathrm{H} 460$ & & & & 0.0221 \\
\hline HPAEpiC & & ORIS was $n$ & $t$ detect & \\
\hline
\end{tabular}

BORIS
Prospective

Average: 0.0071 FPKM
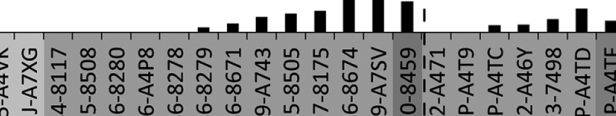

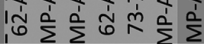

Remission

Progressive Partial remission

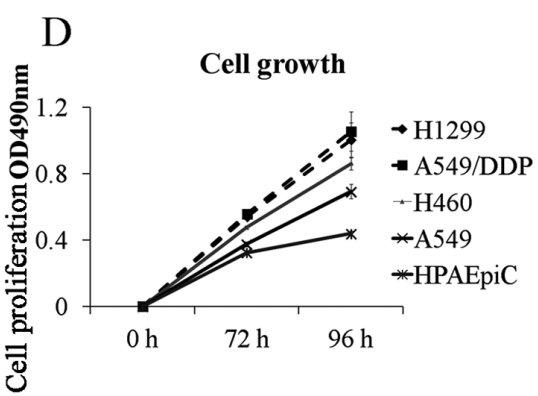

Figure 1. BORIS expression is associated with overall survival rate in patients with NSCLC who received DDP chemotherapy. (A) Overall survival rate curves in patients with NSCLC who received DDP chemotherapy. The data were analyzed using SPSS Kaplan-Meier. Statistical difference was tested using the Log-rank (MANTEL-COX) test. (B) BORIS expression in 23 patients, who had records of treatment outcomes in The Cancer Genome Atlas. The patients were featured according to the outcomes following DDP treatment: Stable disease after DDP chemotherapy, patients completely remitted or responded to DDP chemotherapy, patients with progressive disease after DDP chemotherapy and a patient who responded partly to DDP chemotherapy. (C) BORIS expressions were compared among four NSCLC cell lines, one normal pulmonary alveolar epithelial cell line (HPAEpiC) and one leukemia cell line which was reported to express BORIS and was used as the positive control for testing BORIS. The statistical differences of BORIS level between cell lines were calculated and shown by P-value in the right panel. The statistical analyses were performed by one-way ANOVA followed Tukey post-hoc test. (D) Relative proliferation rates of 4 lung cancer cell lines as compared with a normal human pulmonary alveolar epithelial cell line (HPAEpiC) at different time points. A549/DDP, DDP resistant A549 cell line; BORIS, Brother of Regulator of Imprinted Sites; DDP, cisplatin; NSCLC, non-small cell lung cancer; OD, optical density; FPKM, fragments per kilobase of exon model per million reads mapped. 
A

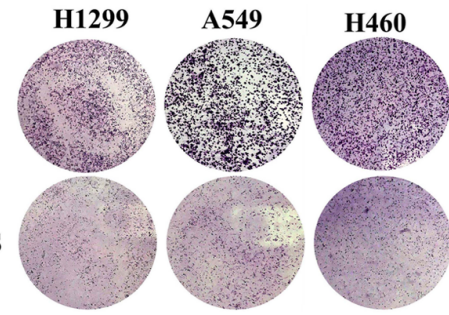

Signal value

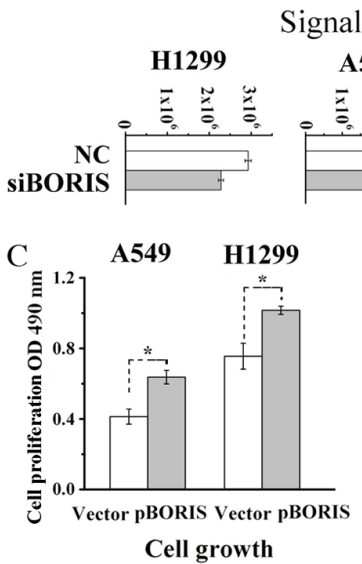

A549

H460

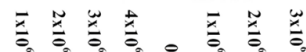

$\mathrm{B}$

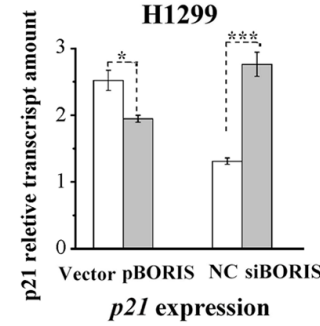

H1299

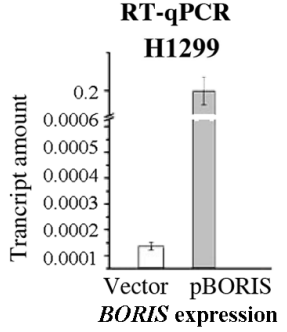

BORIS expression
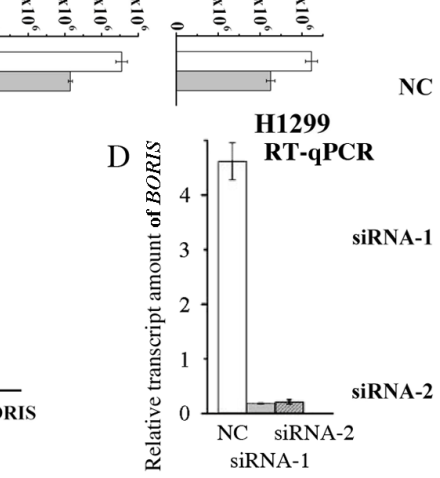

SIRNA-1
SiRNA-2
2 DAPI BORIS $\gamma \mathrm{H} 2 \mathrm{AX}$ Merged

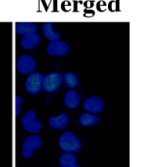

H1299

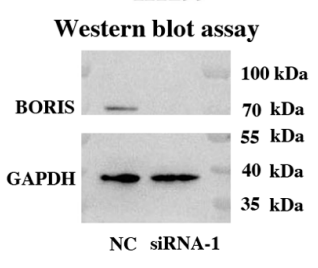

Figure 2. BORIS promotes NSCLC cell proliferation. (A) BORIS knockdown suppressed colony formation of NSCLC cells. Crystal violet was used to stain the cells in upper panels. The bottom graphs present the signal difference in the upper panels. (B) Expression of $p 21$ regulated by BORIS detected by RT-qPCR. The overexpression efficiency of pBORIS was assessed via RT-qPCR analysis (right panel). (C) BORIS overexpression promoted cell proliferation of A549 and H1299 cells measured by MTT assay. (D) Knockdown of BORIS by either siRNA-1 or siRNA-2 induced DNA damage efficiently measured by immunofluorescence stain of $\gamma \mathrm{H} 2 \mathrm{AX}$ (Red). BORIS proteins were detected by the primary BORIS antibody (FITC). The right and left panels presented transfection efficiencies of BORIS siRNA by western blot and RT-qPCR. "P<0.05; ${ }^{* * *} \mathrm{P}<0.001$. A549/DDP, DDP resistant A549 cell line; BORIS, Brother of Regulator of Imprinted Sites; NSCLC, non-small cell lung cancer; OD, optical density; RT-q, reverse transcription-quantitative; si, small interfering; nc, negative siRNA control.

knockdown induced $\gamma \mathrm{H} 2 \mathrm{AX}$ (Fig. 2D), suggesting that BORIS deficiency induces DNA damage in NSCLC cells. Collectively, these data demonstrated that BORIS expression was associated with NSCLC cell proliferation, indicating that BORIS may protect lung cancer cells from DNA damage.

BORIS resists DDP induced cell suppression and apoptosis. In order to determine whether BORIS contributes to DDP resistance, BORIS was first silenced or overexpressed in H1299 cells, and subsequently treated by DDP at various concentrations from 1-4 $\mu \mathrm{g} / \mathrm{ml}$. BORIS knockdown inhibited H1299 cell proliferation. The supplement of 1 or $2 \mu \mathrm{g} / \mathrm{ml}$ DDP synergized BORIS knockdown for cell proliferation suppression (Fig. 3A). Conversely, BORIS overexpression promoted cell proliferation. BORIS overexpression resisted $1-4 \mu \mathrm{g} / \mathrm{ml}$ DDP treatment (Fig. 3B). Subsequently, the extent of apoptosis which was induced by $2 \mu \mathrm{g} / \mathrm{ml}$ DDP treatment was investigated in BORIS overexpressed H1299 cells compared with the negative control. The results demonstrated that BORIS overexpression inhibited DDP induced apoptosis from 13.41 to $8.68 \%$, and necrosis from 5.60 to $3.46 \%$ (Fig. 3C). Taken together, these results suggest that BORIS protects NSCLC cells from injury by DDP treatment, thus, confirm the importance of BORIS in DDP resistance.

BORIS suppresses DNA damage and activates the DNA repair system. As BORIS resisted DDP-induced NSCLC suppression (Fig. 3), it was proposed that BORIS may inhibit DDP-induced DNA damage. pBORIS plasmid (BORIS-flag) was transiently transfected in A549 cells that were treated subsequently by DDP to induce DNA damage. Cells with overexpression of BORIS demonstrated a decreased $\gamma \mathrm{H} 2 \mathrm{AX}$ signal (Fig. 4A and B), indicating that BORIS attenuated DDP-induced DNA damage, thus promoting the proliferation of NSCLC. DNA damage was detected by the TUNEL assay in H1299 and A549/DDP when BORIS were silenced (Fig. 4C). BORIS may be beneficial for the DNA repair system of NSCLC cells to sustain genome stability under the treatment of DDP. The expression levels of few representative genes of DNA repair system were investigated, such as BRCA1, ERCC1, CMYC and MSH6 (Table SII). BORIS was overexpressed by pBORIS or silenced by siRNA-1 (siBORIS in Fig. 4D) in H1299 and H460 cells. Fold changes of the investigated genes are presented in Fig. 4D. MSH6 was regulated consistently and significantly by BORIS in H1299 and H460 cells (Fig. 4D). Collectively, the results of the present study suggest that BORIS may enhance the mismatch repair (MMR) system in NSCLC cells to resist DDP chemotherapy.

\section{Discussion}

The results of the present study demonstrated the association between BORIS expression and DDP resistance in NSCLC. As P53 mutants have frequently been detected in different types of cancer and were reported to account for DDP resistance (25), the present study investigated the effects of BORIS knockdown in H1299 (lack of p53) and A549 (express wild type p53) cells, respectively (19). Suppression of cell viability on BORIS knockdown in both H1299 and A549 cells suggested that the 


\section{A Transfected H1299 treated by DDP for $72 \mathrm{~h}$}

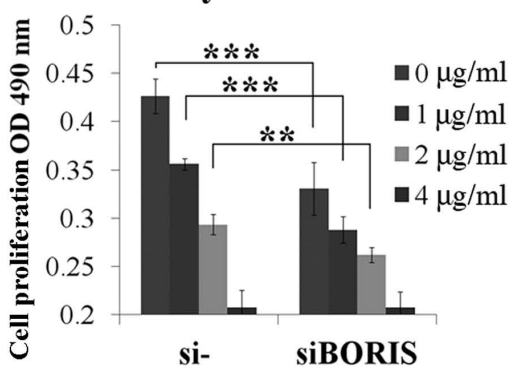

B Transfected H1299 treated by DDP for $72 \mathrm{~h}$

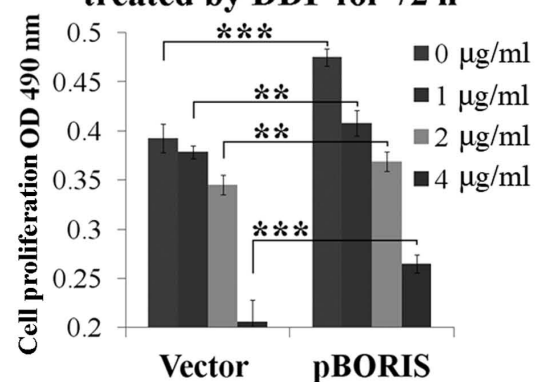

\section{H1299 with $2 \mu \mathrm{g} / \mathrm{ml}$ DDP treatment}
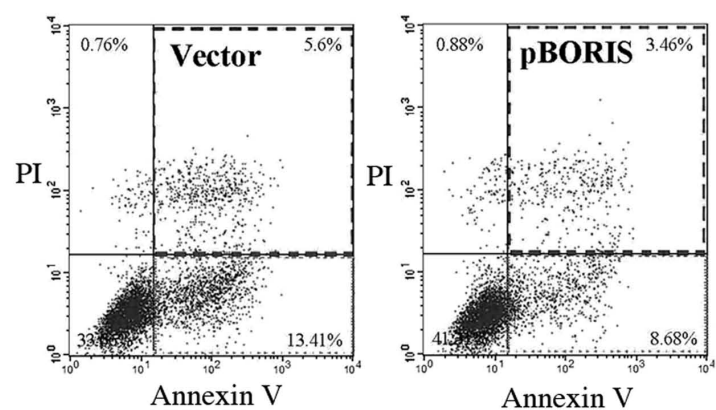

Percentage of total $(\%)$

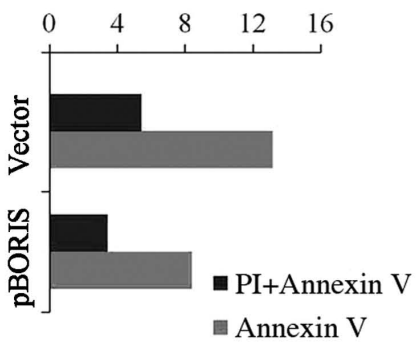

Figure 3. BORIS resists DDP-induced tumor cell suppression. (A) Cell proliferation study of H1299 cells transfected by NC (negative siRNA control) or siBORIS (BORIS siRNA-1) for $24 \mathrm{~h}$ and subsequently treated by DDP at different doses $(0,1,2$ and $4 \mu \mathrm{g} / \mathrm{ml})$ for $72 \mathrm{~h}$. (B) Cell proliferation study of H1299 cells transfected by the vector (empty plasmid) or pBORIS (BORIS overexpression plasmid) for $24 \mathrm{~h}$ and subsequently treated by DDP $(0,1,2 \mathrm{and} 4 \mu \mathrm{g} / \mathrm{ml})$ for $72 \mathrm{~h}$. (C) Cell apoptosis of H1299 cells with overexpression of BORIS after treatment using $2 \mu \mathrm{g} / \mathrm{ml}$ DDP for $24 \mathrm{~h}$. BORIS overexpression suppressed DDP-induced apoptosis in H1299 cells. ${ }^{* *} \mathrm{P}<0.01 ;{ }^{* * * *} \mathrm{P}<0.001$. BORIS, Brother of Regulator of Imprinted Sites; NSCLC, non-small cell lung cancer; OD, optical density; si, small interfering; PI, propidium iodide; DDP, cisplatin.

A

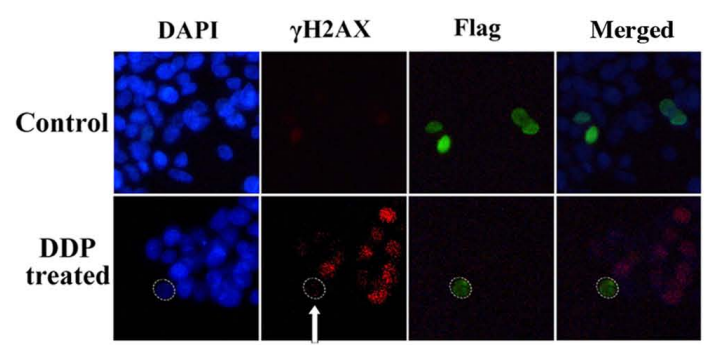

C
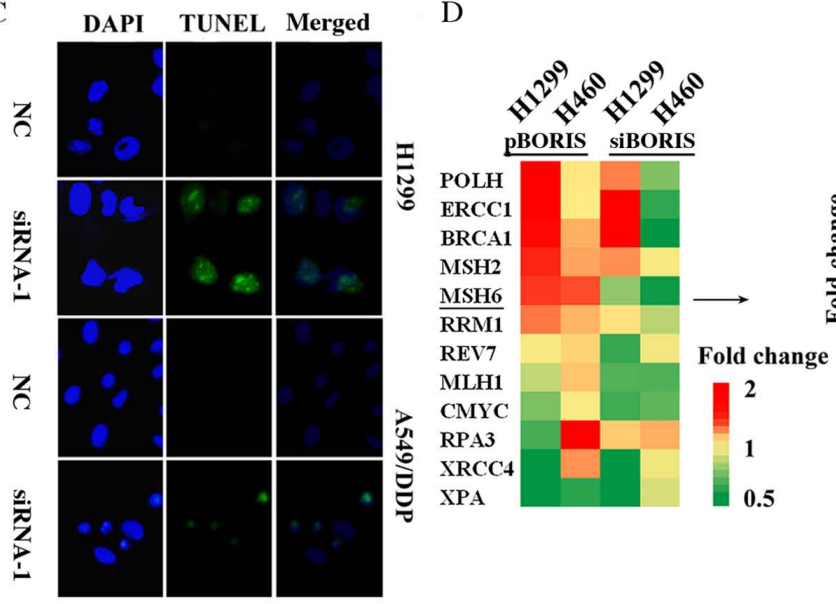

B

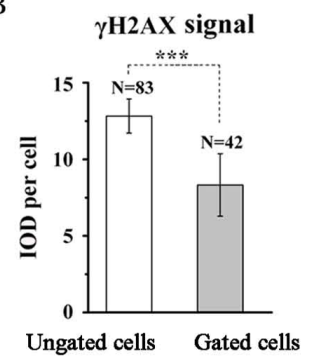

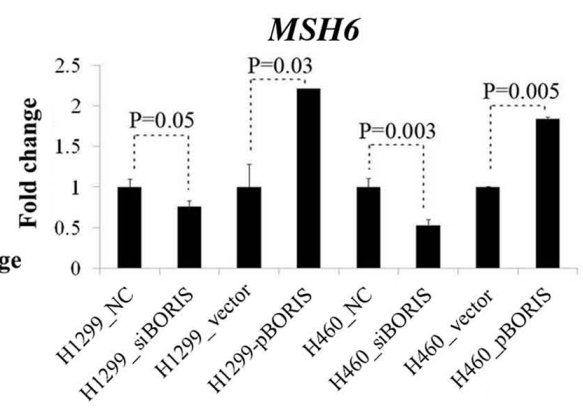

Figure 4. BORIS resists DDP-induced DNA damage. (A) Immunofluorescence stain of H1299 cells transfected by pBORIS (BORIS-flag) and then treated by $1 \mu \mathrm{g} / \mathrm{ml}$ DDP for $24 \mathrm{~h}$. BORIS expression was detected by flag antibody and FITC conjugated secondary antibodies (green). $\gamma \mathrm{H} 2 \mathrm{AX}$ antibody and TRITC conjugated secondary antibodies were used to detected DNA damage (red). (B) Quantification of immune fluorescence signals inside the dotted cycles in panel A. The column demonstrates the $\gamma \mathrm{H} 2 \mathrm{AX}$ (DNA damage) signal difference between gated (BORIS-flag transfected) and ungated cells. (C) H1299 and A549/DDP cells were transfected by BORIS siRNA-1. The knockdown of BORIS induced TUNEL signals in both cell lines. (D) H1299 and H460 cells were transfected by pBORIS or BORIS siRNA-1. Expression levels of the DNA repair associated genes were tested by reverse transcription-quantitative PCR. $M S H 6$ was regulated by the treatments. ${ }^{* * * *} \mathrm{P}<0.001$. IOD, integral optical density; A549/DDP, DDP resistant A549 cell line; BORIS, Brother of Regulator of Imprinted Sites; NSCLC, non-small cell lung cancer; OD, optical density; si, small interfering; nc, negative siRNA control; MSH6, mutS homolog 6. 
Table II. Logistic regression analysis of 2-year survival and predictors in patients with NSCLC.

\begin{tabular}{|c|c|c|c|c|c|c|}
\hline \multirow[b]{2}{*}{ Variable } & \multicolumn{3}{|c|}{ Univariate analysis } & \multicolumn{3}{|c|}{ Multivariate analysis ${ }^{\mathrm{a}}$} \\
\hline & OR & $95 \% \mathrm{CI}$ & P-value & OR & $95 \% \mathrm{CI}$ & P-value \\
\hline \multicolumn{7}{|l|}{ Surgery method } \\
\hline No surgery & Reference & & & Reference & & $<0.001$ \\
\hline Local tumor destruction & 0.18 & $0.16-0.20$ & $<0.001$ & 0.29 & $0.26-0.32$ & $<0.001$ \\
\hline Wedge or segmental resection & 0.10 & $0.09-0.12$ & $<0.001$ & 0.15 & $0.13-0.16$ & $<0.001$ \\
\hline Lobectomy & 0.15 & $0.13-0.16$ & $<0.001$ & 0.13 & $0.11-0.15$ & $<0.001$ \\
\hline Liver transplantation & 0.05 & $0.05-0.06$ & $<0.001$ & 0.08 & $0.07-0.10$ & $<0.001$ \\
\hline Tumor size, mm & 1.02 & $1.02-1.02$ & $<0.001$ & 1.01 & $1.01-1.01$ & $<0.001$ \\
\hline Age, years & 1.02 & $1.02-1.02$ & $<0.001$ & 1.01 & $1.01-1.02$ & $<0.001$ \\
\hline Education level & 1.01 & $1.01-1.02$ & $<0.001$ & 0.98 & $0.98-0.99$ & $<0.001$ \\
\hline Family income & 0.99 & 0.99-0.99 & $<0.001$ & 0.99 & 0.99-0.99 & $<0.001$ \\
\hline \multicolumn{7}{|l|}{ Historic stage } \\
\hline Localized & Reference & & & Reference & & \\
\hline Regional & 5.78 & $5.34-6.28$ & $<0.001$ & 2.32 & $1.97-2.73$ & $<0.001$ \\
\hline Distant & 1.04 & $0.98-1.11$ & 0.202 & 1.09 & $0.98-1.22$ & 0.105 \\
\hline \multicolumn{7}{|l|}{ ACJJ_T } \\
\hline $\mathrm{T} 1$ & Reference & & & Reference & & \\
\hline $\mathrm{T} 2$ & 1.17 & $1.09-1.26$ & $<0.001$ & 1.21 & $1.10-1.33$ & $<0.001$ \\
\hline $\mathrm{T} 3$ & 5.50 & $5.06-5.97$ & $<0.001$ & 1.77 & $1.58-1.98$ & $<0.001$ \\
\hline $\mathrm{T} 4$ & 7.00 & $5.78-8.54$ & $<0.001$ & 1.66 & $1.31-2.12$ & $<0.001$ \\
\hline \multicolumn{7}{|l|}{ Diagnosis year } \\
\hline 2004-2008 & Reference & & & Reference & & \\
\hline 2009-2012 & 0.88 & $0.82-0.94$ & $<0.001$ & 0.72 & $0.66-0.79$ & $<0.001$ \\
\hline 2013-2015 & 0.57 & $0.54-0.61$ & $<0.001$ & 0.48 & $0.44-0.53$ & $<0.001$ \\
\hline \multicolumn{7}{|l|}{ Grade } \\
\hline $\mathrm{I}$ & Reference & & & Reference & & \\
\hline II & 0.97 & $0.90-1.06$ & $<0.001$ & 1.30 & $1.18-1.43$ & $<0.001$ \\
\hline III & 2.22 & $2.01-2.45$ & $<0.001$ & 2.44 & $2.15-2.76$ & $<0.001$ \\
\hline IV & 3.34 & $2.50-4.51$ & $<0.001$ & 3.68 & $2.59-5.29$ & $<0.001$ \\
\hline ACJJ_M & 2.04 & $1.89-2.21$ & $<0.001$ & & & \\
\hline M0 & Reference & & & Reference & & \\
\hline M1 & 11.69 & $10.14-13.55$ & $<0.001$ & 1.74 & $1.33-2.27$ & $<0.001$ \\
\hline
\end{tabular}

function of BORIS was not associated with p53. BORIS may promote DDP resistance by upregulating MSH6 expression. However, the underlying molecular mechanism as to how BORIS regulates MSH6 remains unknown. BORIS was reported to regulate $C M Y C$ expression by demethylating the promoter (26). Demethylation may be a potential mechanism for regulating MSH6 or other genes in the DNA repair system by BORIS to resist DDP treatment. Furthermore, unknown cellular environments may be constructed by BORIS in cancer cells to resist chemotherapy. In neuroblastoma, BORIS was reported to be upregulated along with the development of ALK inhibition resistance (27). It is speculated that BORIS expression may promote cancer cells to resist multiple drug treatments, including DDP. It is well-known that EGFR mutants cause tyrosine kinase inhibitors (RTKIs) resistance in
NSCLC $(28,29)$, thus, it is worth investigating whether BORIS knockdown attenuates RTKIs resistance.

BORIS knockdown induced DNA damage and $p 21$ expression in NSCLC cells. As BORIS is only expressed in cancer cells and testes (30), BORIS may be a key protector for cancer cell genome stability. BORIS is the paralogue of CTCF and is speculated to compete with CTCF to induce the expression of the oncogene (31-33). The spatial genome structure organized by CTCF may be interfered by BORIS (30,34-36). DNA and histone methylation modulated by BORIS may also promote genome stability $(26,37,38)$.

The overexpression of BORIS sustained DDP-induced apoptosis and repaired DNA damage in NSCLC cells. DDP resistance is usually associated with the increased repair of DNA damage recognized by the mismatch repair 
system $(13,14,39)$. MutSa (composed of MSH2 and MSH6) recognized DNA lesions formed by DDP and subsequently recruited downstream MMR proteins, including MutL $\alpha$ (MLH1-PMS2), Exonuclease I, DNA polymerase $\delta$ and DNA ligase $(14,40)$. The elevated expression of MSH6 induced by BORIS overexpression in the present study could facilitate the recognition of DNA lesions and attenuate the recruitment of the phosphorylated form of $\gamma \mathrm{H} 2 \mathrm{AX}$ to sites of DNA damage (24).

In conclusion, BORIS is required for genome stability of NSCLC cells and is prospective therapeutic target for decreasing DDP resistance. Considering that RTKIs resistance is frequent in lung cancer cells, further study of targeted therapy on BORIS will be expected in RTKIs resistant lung cancer cells.

\section{Acknowledgements}

The authors would like to thank Mr. Xiaoliang Zheng, Ms Dongmei Yan, Mr. Jing Jia and Ms Jie Yuat the Center for Molecular Medicine (Hangzhou, China) for their technical assistance and discussion surrounding the data. The authors would also like to thank Professor Tianhui Chenfrom Zhejiang Cancer Hospital (Hangzhou, China) for revising the manuscript.

\section{Funding}

The present study was funded by grants from the National Natural Science Foundation of China (grant no. 31871393); Zhejiang Provincial Natural Science Foundation of China (grant nos. LQY18H300001 and LQ18C070002); Youth Foundation of Zhejiang Academy of Medical Sciences (grant nos. 2019Y006 and 2019Y003); and the Medical and Health Science and Technology Project of Zhejiang Province (grant nos. 2017KY308 and 2019RC030).

\section{Availability of data and materials}

The datasets used and/or analyzed during the present study are available from the corresponding author upon reasonable request.

\section{Authors' contributions}

YS and YZ designed the experiments. YS and CL performed the cell culture, drug treatment experiments and collected the data. JR performed extraction and gene expression analysis. MF performed the plasmid construction. YS and JF performed the statistical analyses. YZ wrote the manuscript. YZ and XW analyzed the data and edited the manuscript. All authors have read and approved the final manuscript.

\section{Ethics approval and consent to participate}

Not applicable.

\section{Patient consent for publication}

Not applicable.

\section{Competing interests}

The authors declare that they have no competing interests.

\section{References}

1. Siegel RL, Miller KD and Jemal A: Cancer statistics, 2016. CA Cancer J Clin 66: 7-30, 2016.

2. Tanaka $\mathrm{F}$ and Yoneda $\mathrm{K}$ : Adjuvant therapy following surgery in non-small cell lung cancer (NSCLC). Surg Today 46: 25-37, 2016.

3. Chevalier TL and Sori JC: Status and trends of chemotherapy for advanced NSCLC. Eur J Cancer Suppl 2: 26-33, 2004.

4. Giaccone G: Clinical perspectives on platinum resistance. Drugs 59 (Suppl 4): S9-S17, S37-S38, 2000.

5. Martin-Kleiner I: BORIS in human cancers-a review. Eur J Cancer 48: 929-935, 2012.

6. Dougherty CJ,Ichim TE, Liu L, Reznik G, Min W, Ghochikyan A, Agadjanyan MG and Reznik BN: Selective apoptosis of breast cancer cells by siRNA targeting of BORIS. Biochem Biophys Res Commun 370: 109-112, 2008.

7. Asano T, Hirohashi Y, Torigoe T, Mariya T, Horibe R, Kuroda T, Tabuchi Y, Saijo H, Yasuda K, Mizuuchi M, et al: Brother of the regulator of the imprinted site (BORIS) variant subfamily 6 is involved in cervical cancer stemness and can be a target of immunotherapy. Oncotarget 7: 11223-11237, 2016.

8. Zhang Y, Fang M, Song Y, Ren J, Fang J and Wang X: Brother of regulator of imprinted sites (BORIS) suppresses apoptosis in colorectal cancer. Sci Rep 7: 40786, 2017.

9. Siddik ZH: Cisplatin: Mode of cytotoxic action and molecular basis of resistance. Oncogene 22: 7265-7279, 2003.

10. Longley DB, Harkin DP and Johnston PG: 5-Fluorouracil: Mechanisms of action and clinical strategies. Nat Rev Cancer 3: 330-338, 2003.

11. Lewin F, Ringborg U, Skog S and Tribukait B: The effect of 5-fluorouracil on cisplatin induced DNA interstrand cross-linking in a mouse ascites tumor growing in vivo. Anticancer Drugs 6: 465-470, 1995.

12. Yoon SL, Roh YG, Chu IS, Heo J, Kim SI, Chang H, Kang TH, Chung JW, Koh SS, Larionov V and Leem SH: A polymorphic minisatellite region of BORIS regulates gene expression and its rare variants correlate with lung cancer susceptibility. Exp Mol Med 48: e246, 2016.

13. Almeida GM, Duarte TL, Farmer PB, Steward WP and Jones GD: Multiple end-point analysis reveals cisplatin damage tolerance to be a chemoresistance mechanism in a NSCLC model: Implications for predictive testing. Int J Cancer 122: 1810-1819, 2008.

14. Shen D, Pouliot LM, Hall MD and Gottesman MM: Cisplatin resistance: A cellular self-defense mechanism resulting from multiple epigenetic and genetic changes. Pharmacol Rev 64: 706-721, 2012.

15. Rosell R, Lord RVN, Taron M and Reguart N: DNA repair and cisplatin resistance in non-small-cell lung cancer. Lung Cancer 38: 217-227, 2002.

16. Cohen SM and Lippard SJ: Cisplatin: From DNA damage to cancer chemotherapy. Prog Nucleic Acid Res Mol Biol 67: 93-130, 2001.

17. Lippard SJ (ed): Platinum, Gold, and OtherMetal Chemotherapeutic Agents. In: Chemistry and Biochemistry. Vol 209. American Chemical Society, 1983.

18. Basu A and Krishnamurthy S: Cellular responses to cisplatin-induced DNA damage. J Nucleic Acids 2010: 201367, 2010.

19. Gomyo Y, Sasaki JI, Branch CD, Roth JA and Mukhopadhyay T: 5-aza-2'-deoxycytidine upregulates caspase-9 expression cooperating with p53-induced apoptosis in human lung cancer cells. Oncogene 23: 6779-6787, 2004.

20. Detterbeck FC, Boffa DJ, Kim AW and Tanoue LT: The eighth edition lung cancer stage classification. Chest 151: 193-203, 2017.

21. Bunz F, Dutriaux A, Lengauer C, Waldman T, Zhou S, Brown JP Sedivy JM, Kinzler KW and Vogelstein B: Requirement for p53 and p21 to sustain G2 arrest after DNA damage. Science 282: 1497-1501, 1998

22. Waldman T, Kinzler KW and Vogelstein B: p21 is necessary for the p53-mediated G1 arrest in human cancer cells. Cancer Res 55: 5187-1590, 1995.

23. el-Deiry WS, Tokino T, Velculescu VE, Levy DB, Parsons R, Trent JM, Lin D, Mercer WE, Kinzler KW and Vogelstein B: WAF1, a potential mediator of p53 tumor suppression. Cell 75: 817-825, 1993. 
24. Mah L, Elosta A and Karagiannis TC: GammaH2AX: A sensitive molecular marker of DNA damage and repair. Leukemia 24: 679-686, 2010.

25. Chee JL, Saidin S, Lane DP, Leong SM, Noll JE, Neilsen PM, Phua YT, Gabra H and Lim TM: Wild-type and mutant p53 mediate cisplatin resistance through interaction and inhibition of active caspase-9. Cell Cycle 12: 278-88, 2013.

26. Nguyen P, Barsela G, Sun L, Bisht KS, Cui H, Kohn EC, Feinberg AP and Gius D: BAT3 and SET1A form a complex with CTCFL/BORIS to modulate H3K4 histone dimethylation and gene expression. Mol Cell Biol 28: 6720-6729, 2008.

27. Debruyne DN, Dries R, Sengupta S, Seruggia D, Gao Y, Sharma B, Huang H, Moreau L, McLane M, Day DS, et al: BORIS promotes chromatin regulatory interactions in treatment-resistant cancer cells. Nature 572: 676-680, 2019.

28. Kobayashi S, Boggon TJ, Dayaram T, Janne PA, Kocher O, Meyerson M, Johnson BE, Eck MJ, Tenen DG and Halmos B: EGFR mutation and resistance of non-small-cell lung cancer to gefitinib. N Engl J Med 352: 786-792, 2005.

29. Lin $Y$, Wang $X$ and Jin H: EGFR-TKI resistance in NSCLC patients: Mechanisms and strategies. Am J Cancer Res 4: 411-435, 2014.

30. Sleutels F, Soochit W, Bartkuhn M, Heath H, Dienstbach S Bergmaier P, Franke V, Rosa-Garrido M, van de Nobelen S, Caesar L, et al: The male germ cell gene regulator CTCFL is functionally different from CTCF and binds CTCF-like consensus sites in a nucleosome composition-dependent manner. Epigenetics Chromatin 5: 8, 2012.

31. Loukinov DI, Pugacheva E, Vatolin S, Pack SD, Moon H, Chernukhin I, Mannan P, Larsson E, Kanduri C, Vostrov AA, et al BORIS, a novel male germ-line-specific protein associated with epigenetic reprogramming events, shares the same 11-zinc-finger domain with CTCF, the insulator protein involved in reading imprinting marks in the soma. Proc Natl Acad Sci USA 99: 6806-6811, 2002.

32. Pugacheva EM, Teplyakov E, Wu Q, Li J, Chen C, Meng C, Liu J, Robinson S, Loukinov D, Boukaba A, et al: The cancer-associated CTCFL/BORIS protein targets multiple classes of genomic repeats, with a distinct binding and functional preference for humanoid-specific SVA transposable elements. Epigenetics Chromatin 9: 35, 2016.
33. Bergmaier P, Weth O, Dienstbach S, Boettger T, Galjart N, Mernberger M, Bartkuhn M and Renkawitz R: Choice of binding sites for CTCFL compared to CTCF is driven by chromatin and by sequence preference. Nucleic Acids Res 46: 7097-7107, 2018.

34. Pugacheva EM, Rivero-Hinojosa S, Espinoza CA Méndez-Catalá CF, Kang S, Suzuki T, Kosaka-Suzuki N, Robinson S, Nagarajan V, Ye Z, et al: Comparative analyses of CTCF and BORIS occupancies uncover two distinct classes of CTCF binding genomic regions. Genome Biol 16: 161, 2015.

35. Lobanenkov VV and Zentner GE: Discovering a binary CTCF code with a little help from BORIS. Nucleus 9: 33-41, 2018.

36. Hong JA, Kang Y, Abdullaev Z, Flanagan PT, Pack SD, Fischette MR, Adnani MT, Loukinov DI, Vatolin S, Risinger JI, et al: Reciprocal binding of CTCF and BORIS to the NY-ESO-1 promoter coincides with derepression of this cancer-testis gene in lung cancer cells. Cancer Res 65: 7763-7774, 2005.

37. Jelinic P, Stehle JC and Shaw P: The testis-specific factor CTCFL cooperates with the protein methyltransferase PRMT7 in H19 imprinting control region methylation. PLoS Biol 4: e355, 2006

38. Sun L, Huang L, Nguyen P, Bisht KS, Bar-Sela G, Ho AS, Bradbury CM, Yu W, Cui H, Lee S, et al: DNA methyltransferase 1 and 3B activate BAG-1 expression via recruitment of CTCFL/BORIS and modulation of promoter histone methylation. Cancer Res 68: 2726-2735, 2008.

39. Gautam A, Li ZR and Bepler G: RRM1-induced metastasis suppression through PTEN-regulated pathways. Oncogene 22: 2135-1242, 2003

40. Martin LP, Hamilton TC and Schilder RJ: Platinum resistance: The role of DNA repair pathways. Clin Cancer Res 14: 1291-1295, 2008.

This work is licensed under a Creative Commons Attribution-NonCommercial-NoDerivatives 4.0 International (CC BY-NC-ND 4.0) License. 